daily was defined as the recommended dose for further studies.

Most encouragingly, objective tumour responses - assessed using the Response Evaluation Criteria in Solid Tumours (RECIST) and ${ }^{18}$ fluorodeoxyglucose positron emission tomography (see picture) - showed that tumour regression occurred in $69 \%$ of patients and, in most patients, within 8 days of beginning treatment. Imatinib was particularly successful in treating patients with advanced GISTs.

So, although still at an early clinical trial stage, imatinib shows great potential for the treatment of this previously intractable tumour. Future studies will hopefully clarify the optimum recommended dosage and duration of treatment.

Sandra Clark

6) References and links ORIGINAL RESEARCH PAPER van Oosterom, A. T. et al. Safety and efficacy of imatinib (STI-571) in metastatic gastrointestinal stromal tumours: a phase I study. Lancet 358, 1421-1423 (2001) FURTHER READING Effect of the tyrosine kinase inhibitor STI571 in a patient with a metastatic gastrointestinal stromal tumor. N. Engl. J. Med. 344, 1052-1056 (2001)

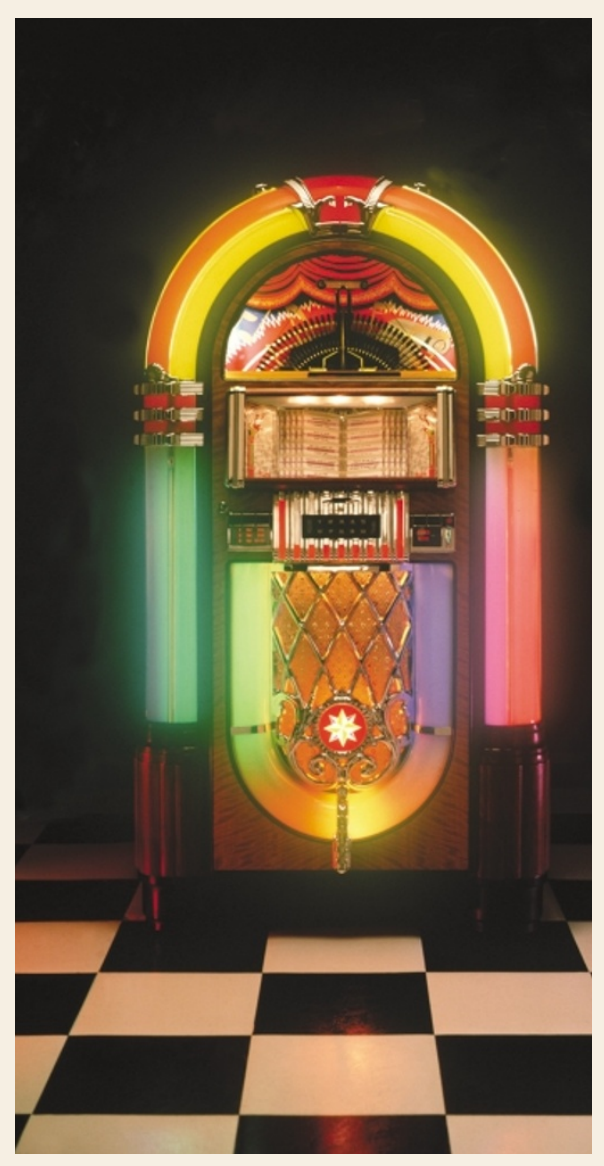

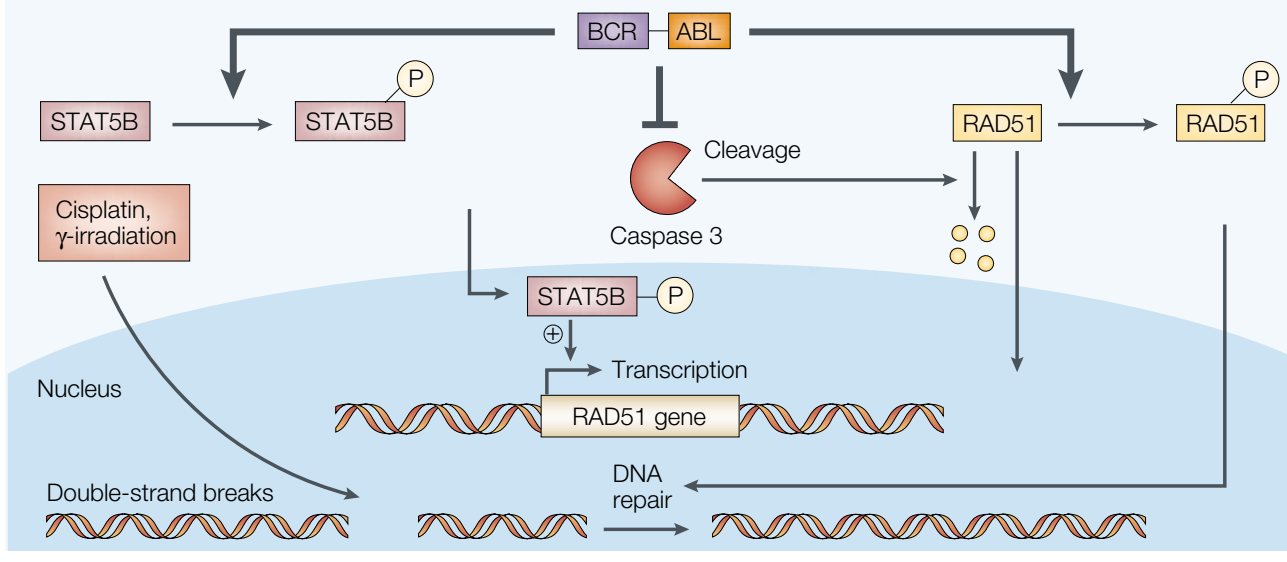

DRUG RESISTANCE

\section{ABL to resist}

We think of cancer cells as having unstable genomes, so it seems counterintuitive that they might increase their ability to repair DNA. But Artur Slupianek and co-workers now describe how some leukaemia cells do just that to resist attack by DNA-damaging drugs.

Tumours expressing oncogenic tyrosine kinases such as $\mathrm{BCR}-\mathrm{ABL}$ - the fusion protein that is a hallmark of chronic myelogenous leukaemia are resistant to DNA-damaging drugs. To find out why, Slupianek and colleagues expressed a deletion mutant of BCR-ABL that lacked the $\mathrm{SH} 2$ and $\mathrm{SH} 3$ domains of $A B L(B C R-A B L \Delta \Delta)$, in a myeloid cell line. Cells expressing this mutant were sensitive to cisplatin and mitomycin $\mathrm{C}$, whereas cells expressing full-length $\mathrm{BCR}-\mathrm{ABL}$ were resistant to these drugs. The $\mathrm{SH} 2$ and $\mathrm{SH} 3$ domains of $\mathrm{ABL}$ are required to activate the transcriptional activator STAT5B, and drug resistance could be reinstated in BCR-ABL $\Delta \Delta$ cells by expressing a dominant-active mutant of STAT5B (STAT5B-DAM). So which of STAT5B's many target genes is responsible for drug resistance? Expression of the DNA-repair gene RAD51 and some of its paralogues was dysregulated in cells expressing either BCR-ABL or the combination of BCR-ABL $\Delta \Delta$ and STAT5BDAM, but not in the parental cell line or in cells overexpressing BCR-ABL $\Delta \Delta$ alone.

Is activation of $R A D 51$ expression controlled directly by STAT5B? STAT5B could drive transcription of a luciferase reporter gene fused to RAD51's promoter in cells expressing $\mathrm{BCR}-\mathrm{ABL}$, but not in cells expressing BCR-ABL $\Delta \Delta$. But increased transcription might not be the whole story: RAD51 is a substrate of the apoptotic protease caspase 3 , which is inhibited by BCR-ABL. Western blots to detect activated fragments of caspase 3 and a proteolytic product of RAD51 revealed that caspase 3 was activated by cisplatin in BCR-ABL $\Delta \Delta$-expressing cells and the parental cell line, but not in cells expressing BCR-ABL. RAD51 overexpression is sufficient to cause drug resistance because expression of $R A D 51$ in BCR-ABL $\Delta \Delta$ cells restored most of their ability to resist cisplatin and mitomycin $C$ treatment, whereas expression of a RAD51 antisense sequence in BCR-ABL-expressing cells sensitized them to the drugs.

Is drug resistance caused by increased ability to repair a lethal accumulation of double-strand breaks, or some other property of RAD51? The repair of double-strand breaks can be measured by transfecting cells with two constructs that, when repaired, yield an intact gene for green-fluorescent protein (GFP) and hence a fluorescent signal. In BCR-ABL-expressing cells, introduction of RAD51 increased levels of repair, whereas a RAD51 antisense construct decreased it. This effect was not seen in cells expressing a kinase-dead mutant of BCR-ABL.

But increasing RAD51 levels is not the only way in which BCR-ABL bolsters DNA repair: coimmunoprecipitations revealed that both $\mathrm{c}-\mathrm{ABL}$ and BCR-ABL interact with RAD51.

Phosphorylation of RAD51 was increased by cisplatin or mitomycin $C$ in the parental cell line, which expresses c-ABL. By contrast, RAD51 was constitutively phosphorylated in cells expressing $\mathrm{BCR}-\mathrm{ABL}$. RAD51 has previously been reported to be phosphorylated by c-ABL on two tyrosine $(\mathrm{Y})$ residues - Y54 and Y315. Tyrosine-tophenylalanine $(\mathrm{F})$ mutations at these two residues indicated that $\mathrm{Y} 315$ is the main site of phosphorylation by BCR-ABL. Transfection of BCR-ABL-positive cells with the Y315F mutant increased their sensitivity to cisplatin and mitomycin $\mathrm{C}$, indicating that phosphorylation of Y315 by BCR-ABL controls drug resistance.

So, BCR-ABL has three different ways of boosting RAD51's activity (see picture): by increasing its expression, decreasing its degradation and activating it through post-translational modification. It's an intriguing possibility that other oncogenic tyrosine kinases might also be able to activate one or more of these mechanisms. Could we resentitize resistant tumours to DNA-damaging agents by treating them with tyrosine kinase inhibitors? And does aberrant expression of RAD51 and its paralogues contribute to genomic instability in malignant cells?

Cath Brooksbank

(4) References and links

ORIGiNAL RESEARCH PAPER Slupianek, A. et al. BCR/ABL

regulates mammalian RecA homologs, resulting in drug resistance. Mol. Cell 8, 795-806 (2001)

WEB SITES

Richard Fishel's lab: http://www.kcc.tju.edu/staff/fishel/

Tomasz Skorski's lab:

http://www.temple.edu/biology/faculty/skorski.html 